\title{
Motion and physiological noise effects on amygdala real-time fMRI neurofeedback
}

\section{learning}

Lydia Hellrung $^{1 *}$, Viola Borchardt ${ }^{2}$, Florian N. Götting ${ }^{2}$, Jörg Stadler ${ }^{3}$, Claus Tempelmann ${ }^{4}$, Philippe N. Tobler ${ }^{1}$,

Martin Walter 2,5, Johan N. van der Meer $2,5,6$

1 Laboratory for Social and Neural Systems Research, Department of Economics, University of Zurich, Zurich, Switzerland

2 Clinical Affective Neuroimaging Laboratory, Department of Behavioral Neurology, Leibniz Institute for Neurobiology, Magdeburg, Germany

3 Special Lab for Non-Invasive Brain Imaging, Leibniz Institute for Neurobiology, Magdeburg, Germany

4 Department of Neurology, Otto-von-Guericke University, Magdeburg, Germany

5 Translational Psychiatry, Department of Psychiatry and Psychotherapy, University Tübingen, Tübingen, Germany

6 QIMR Berghofer Medical Research Institute, Brisbane, Australia

\section{Corresponding authors:}

Lydia Hellrung, Laboratory for Social and Neural Systems Research, Department of Economics, University of Zurich, Blümlisalpstrasse 10, 8006 Zurich, Switzerland; E-mail: lydia.hellrung@econ.uzh.ch

Johan van der Meer, QIMR Berghofer Medical Research Institute, Systems Neuroscience, 300 Herston Rd 4006 Brisbane, QLD, Australia; E-mail: Johan.vanderMeer@qimrberghofer.edu.au; 


\section{Abstract}

Real-time fMRI neurofeedback allows to learn control over activity in a localized brain region. However, with fMRI, physiological factors such as the cardiac cycle and respiration interfere with the measurement of brain activation. In conventional fMRI studies this is usually mitigated by inclusion of motion parameters and/or physiological parameters as nuisance regressors at the analysis stage, allowing to correct for and filter out such confounders. In real-time fMRI, however, such an approach is not routinely feasible due to the necessity to process all signals during the runtime of an experiment. The absence of on-line correction can therefore compromise realtime fMRI study outcomes reporting volitional self-regulation capability as BOLD signal changes. This is especially true for BOLD signal changes in subcortical regions situated close to blood vessels or air cavities, such as the amygdala. We therefore aimed to establish the effects of motion, heart rate, heart rate variability, and respiratory volume on learning effects, which means here an increase in BOLD signal change over NF training, in an amygdala neurofeedback experiment. Specifically, we investigate motion parameters from two emotion regulation studies - performed at $3 \mathrm{~T}$ and $7 \mathrm{~T}$ scanners - and additionally acquired physiological variance for the latter one. Our results revealed differences in these parameters between groups and especially between regulation and resting periods within each participant. However, strictly considering these parameters as nuisance regressors in data analysis revealed that the learning of volitional self-regulation of the amygdala is not driven by motion and physiological changes. As validation of our real-time findings, we compare them to the gold standard of assessment of motion and physiology from the Human Connectome Project. Based on this, we recommend to carefully report neurofeedback study results including physiological nuisance regression. To our knowledge, this is the first study investigating the effects of motion and physiological noise correction on neurofeedback BOLD effects.

Keywords: real-time fMRI, ultra-highfield fMRI, neurofeedback, amygdala, physiological noise correction, motion correction 


\section{$1 \quad 1$ Introduction}

2 Real-time fMRI (rt-fMRI) allows to learn volitional self-regulation and has been performed with numerous brain

3 regions (Sitaram et al., 2016). A region of interest (ROI) is determined via either an anatomical or a functional

4 localizer, which is subsequently used to extract the blood oxygenation level dependent (BOLD) signal for

5 neurofeedback (NF). The presence of the neurofeedback signal thereby allows participants to exert control over

6 BOLD activity through training. Unfortunately, BOLD activity changes can also be induced by motion or

7 physiological changes, such as changes in heart rate and/or respiration (Kasper et al., 2017; Parkes et al., 2018).

8 Respiration and heart rate could impact BOLD in two ways - first by pulmonary and respiratory motion interacting

9 with the localized magnetic field distortion causing signal distortion and dropout; second by changing the blood

10 oxygenation levels all over the brain, impacting the BOLD contrast at a physiological level. To clearly separate

11 NF learning effects from such confounds requires to fully consider these confounds during data acquisition and

12 analysis. So far, in conventional fMRI research head motion can be extracted from imaging data and respiration

13 and the pulsation of blood vessels can be acquired in parallel to imaging with appropriate sensors (respiration belt

14 for breathing and electrocardiogram (ECG) or pulse oximeter (PPU) for heart beat). Nowadays, this is a highly-

15 recommended procedure. Taking these data into consideration for analysis is straightforward with the appropriate

16 toolboxes, such as the PhysIO Toolbox (Kasper et al., 2017). These toolboxes extract nuisance parameters from a

17 complete data set. The extracted parameters can then be used for offline fMRI data analysis to regress out motion

18 and physiology-related variance in the BOLD signal.

For rt-fMRI it is more challenging to properly consider such nuisance variables due to the component of the on-

line analysis, with (by necessity) reduced amount of available data points during the runtime of an experiment.

The effects of motion in on-line analysis are usually considered in terms of an on-line realignment of the image data. Such approaches are either already provided by the sequence reconstruction from the MR scanner (e.g. Siemens or Philips prospective motion correction for EPI imaging) or by on-line preprocessing within the software processing pipeline (Goebel, 2012; Hellrung et al., 2015; Koush et al., 2017a). It is challenging to include rotation and translation parameters in rt-fMRI in analogy to conventional fMRI. However, some software toolboxes allow 
et al., 2015; Hinds et al., 2011; Koush et al., 2017b). In contrast, real-time correction of physiological confounders is currently not possible and it still is a largely open question how they affect neurofeedback results.

Therefore, in this study, we investigate the effects of motion, heart rate variability and respiration on the NF

learning effects in rt-fMRI regulation experiments. The amygdala is a ROI of high interest for rt-fMRI neurofeedback experiments. Its capacity for being modulated by means of neurofeedback has been investigated so far in numerous studies including two own previous studies (Brühl et al., 2014; Hellrung et al., 2018; Marxen et al., 2016; Paret et al., 2014; Zotev et al., 2011). Furthermore, amygdala NF training has been proposed as a potential complement to pharmacological interventions for disorders, such as depression (Young et al., 2014) and Posttraumatic Stress Disorder (Gerin et al., 2016). However, the effects of motion, respiration and heart rate could be especially profound for the amygdala due to its close proximity to major blood vessels (Boubela et al., 2015), and its proneness for signal dropout (Deichmann et al., 2002).

Specifically, we need to know how head motion, respiration and heart beat will affect real-time fMRI learning effects, especially for amygdala. The following questions need to be answered:

(1) How do motion and physiological measures (heart rate, heart rate variability and respiration) vary during the neurofeedback experiment, and does they vary as a function of either intervention group (feedback vs. no feedback) or actions within the experiment (rest vs. regulation)?

(2) Do these parameters correlate with learning effects, i.e. increase together with increased capacity of BOLD regulation across several training runs?

To answer these questions, we acquired data in a real-time amygdala neurofeedback experiment paying special attention to motion and using respiration belt and pulse oximetry to assess respiration and heart rate, respectively. Moreover, we aimed to test whether the variations within our real-time data fall within the normally expected range from a big sample. To do so, we used motion, respiration and heart rate data from the human connectome project which provides a gold standard for quantifying variability in these parameters. The results of our study 
speak to the growing NF literature. Importantly, our data clearly indicate that amygdala NF learning effects are not primarily driven by motion and physiological noise, a finding which reinforces the feasibility and reliability of amygdala self-regulation. However, our results also illustrate the necessity to report in detail how rt-fMRI studies control for motion and physiological parameters in the analysis.

\section{Methods}

\subsection{Participants}

At the 7 Tesla site in Magdeburg, 34 participants were recruited to perform either an intermittent NF (7T INT; $\mathrm{N}=20$ ) or a no feedback version (7T NOF; $\mathrm{N}=14$ ) task. At the 3 Tesla site in Leipzig, a total of 42 participants were recruited to perform a continuous NF (3T CON; $\mathrm{N}=18$ ), intermittent NF (3T INT; $N=16)$, or a no feedback version (3T NOF; N=8) task. At both sites, the same experimental NF task has been performed (see 2.3). All procedures were conducted in accordance with the Declaration of Helsinki and were approved by the Ethics Committees of the Faculties of Medicine of the Universities of Magdeburg and Leipzig for the 7T and 3T data acquisition, respectively. Participants gave informed written consent prior to participating in the study.

\subsection{Experimental procedure MR acquisition}

MRI data acquisition: Scanning was performed using 12-channel head coil on 3T scanner and a 32-channel head coil on 7T. For functional imaging, the repetition time (TR) was identical (2s) on both scanners to facilitate an identical stimulus presentation. In the 7T experiment, the following parameters were used for functional imaging: echo time $(\mathrm{TE})=20 \mathrm{~ms}$; matrix size $=160 \times 160$ voxel; bandwidth $=1838 \mathrm{~Hz}$, flip angle $=90^{\circ}$; voxel size $=$ $1.4 \times 1.4 \times 1.8 \mathrm{~mm}^{3}$, GRAPPA factor $=3$. The used sequence served to corrected for motion and distortion during the reconstruction of the images (In and Speck, 2012). These data have been used for the real-time calculation of the feedback signal. In addition, the sequence provided uncorrected data, which has been used for motion analysis within this study. For 3 Tesla, the following parameters were used for functional imaging: TE $=25$ ms; matrix size $=64 \times 64$; bandwidth $=1953 \mathrm{~Hz}$; flip angle $=90^{\circ}$; voxel size: $3 \times 3 \times 2.6 \mathrm{~mm}^{3}$. Here, the real-time motion correction has been applied by our analysis toolbox after data export to the external analysis computer. Prior to the 
functional imaging in both experiments, T1-weighted images were acquired for anatomical normalization using three-dimensional magnetization-prepared rapid gradient echo (MPRAGE) sequence (sagittal orientation) with selective water excitation and linear phase encoding (Mugler and Brookeman, 1990).

Amygdala Region of Interest (ROI) Delineation: The T1 scan was used to demarcate manually the left amygdala.

Amygdala masks were drawn individually per participant into T1 data by a neurologist using FSLview ${ }^{1}$ (Jenkinson et al., 2012). The amygdala ROI was co-registered and re-sampled into the participant's own functional EPI space by performing a short localizer functional scan, co-registering this scan to the participant's T1 scan, and then applying the inverse transformation on the amygdala mask.

Real-time Data transfer and fMRI Neurofeedback Software: The neurofeedback setup at both sites was as previously reported in our study (Hellrung et al., 2018). In short. We were using the in-house toolbox rtExplorer (Hollmann et al., 2011, 2008) and a direct transfer of the data via network connection. The data were sent volumewise to a network port and stored into the random-access memory of the analysis computer. For 3T, data were motion corrected using the preprocessing module of the in-house software BART (Hellrung et al., 2015), while 7T data were motion corrected during the reconstruction within the MR sequence.

\section{Physiological Recordings:}

92 At the 7T site, we additionally acquired respiration information with a respiration belt (using a pneumatic respiration transducer from Honeywell 40PC001B1A) and heart-rate information with a NONIN (8600-FO) pulse oximeter on the right index finger. For digital recording and subsequent analysis of physiological data we used an in-house setup, consisting of the hardware "PhysioBox" and the software "Physiolog"2. The PhysioBox employs the National Instruments acquisition card USB 6008. The Software "Physiolog" written in Python samples the data at $200 \mathrm{~Hz}$ and stores them as CSV

\footnotetext{
1 http://fsl.fmrib.ox.ac.uk/

2 https://cni.lin-magdeburg.de/index.php/en/wiki/mri/physiological-data/
} 
parameters have been acquired. Our questions upon physiological confounders arose from the

100 experiences we gained from that experiment.

\subsection{Experimental neurofeedback paradigm}

102 The NF paradigm required participants to generate a positive mood by remembering positive memories during up103 regulation (HAPPY) blocks. During counting (COUNT) blocks, participants were requested to count backwards

104 from 100 in steps of 3. During resting (REST) blocks, participants were requested to disengage from the task. All 105 blocks lasted 40 seconds. The three different blocks were prompted by different cues: a red arrow pointing upwards 106 for HAPPY, a blue arrow pointing downwards for COUNT, and a white cross for REST. A thermometer was present 107 during each block and was updated in a different fashion for each experimental group as follows: For the 108 intermittent neurofeedback group (INT), the thermometer remained blank during the HAPPY and COUNT blocks,

109 but was updated at the end for 4 seconds with a value representing the average difference in image intensity 110 between the regulation block (HAPPY or COUNT) and the preceding REST block. For the NOF group, the 111 thermometer remained blank in this time period. Participants of the INT group were instructed to attempt to 112 maximally increase (HAPPY) or maximally decrease (COUNT) the thermometer display. During REST the 113 thermometer remained still at the zero point.

The 40-second blocks of REST, HAPPY and COUNT were repeated 4 times in a single run lasting 8 minutes and 40 seconds (with an additional REST block at the end). In the INT group, this resulted in 8 presentations of the

117 NF information, here, i.e. the current BOLD activity within the amygdala. This information was presented at the end of each regulation block ( 4 for HAPPY, 4 for COUNT) lasting for $4 \mathrm{~s}$, which facilitate NF training. The complete rt-fMRI NF experiment consisted of three such training runs. Preceding the training runs, participants performed a Baseline practice run, which consisted only of four HAPPY runs of $80 \mathrm{~s}$ each, interleaved with 40

121 seconds REST, cued with a red upward arrow and concluded with a 4s presentation of NF information. The

122 Baseline enabled participants to practice up-regulation strategies and get used to the scanner environment. The 123 training runs were followed by a Transfer run, which was identical to the Training runs but did not present any NF 124 information. 
125 Before the experiment commenced, a short 40 -second localizer functional scan was performed to enable coregistration of the previously delineated Amygdala Mask into the current functional EPI space.
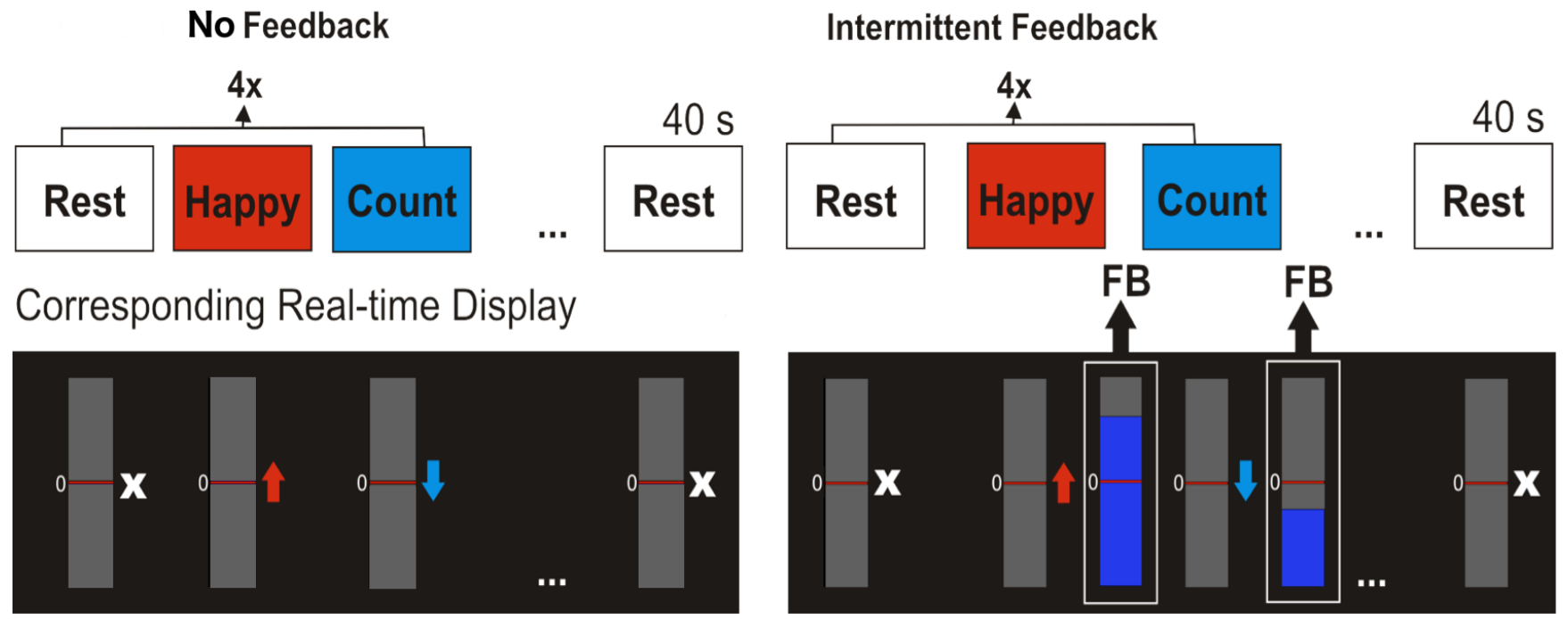

Fig. 1 NF paradigm for amygdala self-regulation: Colored arrows or a white cross indicated the current regulation block. The intermittent group (INT) received feedback about their current amygdala activity every $40 \mathrm{~s}$ at the end of the regulation block. The control group (NOF) did not receive any feedback throughout the experiment. Overall, the participants repeated this paradigm in three training runs and one transfer run without any feedback.

\section{$127 \quad 2.4$ Validation with HCP data}

128 To gauge our levels of variability in motion and physiology against those normally found during MR acquisition, we compared our data to those of the Human Connectome Project (HCP). We used a mirror of the HCP data stored on Australia's high-performance computing infrastructure located in Melbourne (MASSIVE) (Goscinski et al.,

131 2014). The HCP data includes cardiac and respiratory signals measured with a pulse oximeter and respiratory

132 bellows (Siemens Physiologic Monitoring Unit). These signals, along with the sync pulse from the scanner, were

133 recorded by the scanner host computer at a sampling rate of $400 \mathrm{~Hz}$.

134 HCP tasks: We chose three different fMRI tasks paradigms from the available datasets: (1) "REST1", where no 135 explicit task was performed, (2) "EMOTION”, which was a block-design emotional picture task supposed to raise 136 amygdala activity and therefore is relevant for comparison to our paradigm, (3) "MOTOR", where participants

137 performed tongue or bilateral finger and feet movements. We chose this task as it is likely to provide an upper 138 level of median framewise displacements and physiological variation. 
139 HCP data: For median framewise displacement evaluation, data of 822 participants were included that had

140 performed all three tasks. To perform between-group statistical comparisons, participants were subdivided into

141 three groups of 274 each, so that each task comprised disjoint participants' data. To estimate variation in

142 physiological parameters, a total of 636 participants were selected for whom physiological recording data were

143 available for all three tasks. These were also divided into three groups, resulting in 212 participants per group.

$145 \quad 2.5$ Data processing and parameter extractions

\section{$146 \quad 2.5 .1 \quad$ Data preprocessing (fMRI)}

147 Preprocessing of the functional data was performed using SPM12 (Wellcome Department of Imaging

148 Neuroscience, London, UK) and Matlab 2011b3. It comprised correction for slice acquisition time within each

149 volume, motion correction (motion correction parameters were extracted and used for further analyses as

150 described below), and co-registration to the T1 scan. Next, DARTEL (Ashburner, 2007) served to normalize

151 functional data to MNI space. Images were resampled with a $1.5 \mathrm{~mm} 3$ voxel size, high-pass filtered with $240 \mathrm{~s}$

152 filter size, and smoothed with a $6 \mathrm{~mm}$ kernel. Further, individual amygdala masks were normalized to the

153 standard MNI template using the individual T1-weighted structural images.

154 2.5.2 Median framewise displacements extraction

155 To quantify and compare motion between participants, we calculated median framewise displacement (MFD)

156 values, which convert the three rotation and three translation parameters obtained from the SPM realignment step

157 in the preprocessing of the functional data into a single motion value per volume (Jenkinson et al., 2002).

158 Specifically, the voxel-specific head motion is a nonlinear combination of the volume-wise translations and 159 rotations, as well as the voxel's position. Here, the voxel-specific movement is handled via a combination of affine

160 matrices for each rotation and translation, $\mathrm{T}_{\mathrm{t}}{ }^{-1}$. The spatial mean across all voxels is then calculated according to 161 Jenkinson:

\footnotetext{
${ }^{3} \mathrm{http}: / /$ www.mathworks.com
} 


$$
M F D_{\text {vol,t }}(\text { Jenkinson })=\sqrt{\frac{1}{5} R^{2} \operatorname{Trace}\left(A^{T} A\right)+(b+A c)^{T}(b+A c)}
$$

163

164

165

166

167

168

169

170

171

172

173

174

175

176

177

178

179

180

181

182

where $\mathrm{R}=80 \mathrm{~mm}$ is assumed as radius of the head, $\mathrm{c}$ indicates the coordinates for the center of the volume and $\mathrm{A}$

and $\mathrm{b}$ are defined as $\left[\begin{array}{ll}A & b \\ 0 & 0\end{array}\right]=T_{t} T_{t-1}^{-1}-I$ defining rotations and translations (equation and further details in Yan et al., 2013a). The calculation here is based on algorithms from the DPARSF toolbox (Chao-Gan and Yu-Feng, 2010a) using Matlab 2011b.

\subsubsection{Physiological parameter extraction}

To assess physiology, for all data sets (HCP data files were downloaded for processing), the signals from the physiology station were converted to input text files for the PhysIO toolbox for analysis and modeling of electrophysiological data (Kasper et al., 2017). This toolbox detects breathing cycles from the respiration data and heart beat markers from the physiological data. For this, template models for the pulse complex and respiration are convolved with the acquired signals to detect peaks as heart beats and volume of breath per given time unit.

Heart Rate / Heart Rate Variability: We calculated the average heart rate (HR in beats/min) and heart rate variability (HRV) within the different tasks (amygdala regulation for NF data and REST1, MOTOR and EMOTION for HCP data): Furthermore, for our data, we split up these values according to our task conditions (HAPPY, COUNT, REST). The HRV was calculated according to the standard deviation of intervals between the single heartbeats:

$$
\mathrm{SDNN}=\sqrt{\frac{1}{N-1} \sum_{j=1}^{N}\left(\mathrm{RR}_{j}-\overline{\mathrm{RR}}\right)^{2}}
$$

where $R R_{j}$ denotes the value of $j$ 'th $R R$ interval and $N$ is the total number of successive intervals.

Respiration: To assess respiration, we used the output of the TAPAS toolbox encoding for respiratory volume per unit time (RVT) (Kasper et al., 2017) per scan. We then calculated the average RVT within the different tasks and conditions of our task. 


\section{$184 \quad$ 2.5.4 Construction of motion and physiological regressors for GLM}

185 To account for motion and physiology in the analyses of the fMRI data, we constructed regressors that can be used 186 to regress out effects from the functional data. In order to project out motion from the fMRI data, we constructed 187 a matrix consisting of columns containing the realignment parameters, the framewise displacement of the 188 realignment parameters, and scan-nulling regressors. The scan-nulling regressors were constructed by the MFD 189 values and applying a threshold of $0.5 \mathrm{~mm}$ to identify functional volumes in which motion exceeded this threshold 190 (Lemieux et al., 2007). A scan-nulling regressor is then constructed for each volume in the interval of -1 to +2

191 before/after that volume. We used the implementation in the covariate regression step of the DPARSF toolbox to 192 implement the formation of the scan nulling regressors (Chao-Gan and Yu-Feng, 2010b). We chose the named 193 definitions of MFD and scan nulling for this study since it has been shown as reliable and valid method in 194 comparison to other comparable approaches (Yan et al., 2013b)

195 To account for physiological confounds, we formed a matrix consisting of 8 regressors. Six were RETROICOR 196 regressors (Glover et al., 2000) to model for cardiac and heart beat phase; we used a first order model for cardiac 197 phase, a first order model for respiratory phase, and a first order interaction model. To account for BOLD variations 198 in the imaging data, these three phase models were expanded using a sine and a cosine function as outlined in the RETROICOR methods paper (REF) and the PhysIO toolbox paper (REF), yielding 6 regressors. In addition to the 6 RETROICOR regressors, we included two additional regressors that directly model for HRV and RVT. The HRV regressor was convolved with the cardiac response function (Chang et al., 2009) and the RVT regressor was convolved with the respiration response function (Birn et al., 2006). This is parallel to the approach as outlined in the PhysIO toolbox (Kasper et al., 2017).

\subsubsection{Amygdala ROI extractions}

205 To assess whether changes over NF training time in the amygdala regulation capability, i.e. the \% signal change 206 in BOLD activity in each training run, could be explained by head motion and/or physiological noise, we 207 constructed three different general linear models (GLM) in SPM12 on single-subject level. All GLMs included 208 eight regressors modelling HAPPY and COUNT conditions for the three training and transfer runs and one 
regressor modelling HAPPY for the Baseline run. The REST condition was treated as implicit baseline, as participants were instructed to disengage from the task and inclusion of this regressor would have led to a singular design matrix. The GLMs differ in the number of nuisance regressors included in the models: (1) without any nuisance regressors (GLM NO NUISANCE), (2) with six motion regressors and scan-nulling regressors as described in 2.5.4 (GLM + MOCO), and (3) with six motion regressors, scan-nulling regressors and eight physiological nuisance regressors as described in 2.5.4 (GLM + MOCO \& PHYSIO). Since physiological data has been acquired for 7T data only, the latter GLM was conducted for 7T dataset only. All single-subject GLMs served to estimate BOLD signal change (\% signal change) of HAPPY vs REST for each run (Baseline, Trainings, Transfer) within the individual amygdala ROI. This was calculated by extracting beta parameter estimates from the GLM result files from the HAPPY condition and the implicit baseline regressor as estimates during REST. We averaged the extracted values from the amygdala ROI mask and divided the averages from HAPPY by those from REST.

\subsection{Statistical analysis}

Median framewise displacements and physiological parameters: We performed statistical comparisons of MFD values, HR, HRV and RVT between the groups (INT, CON), condition-wise (HAPPY, COUNT, REST), and within-subjects along the runs (Baseline, Run1, Run2, Run3, Transfer) to assess the dynamics of motion. We were using ANOVA functions for between- and within-subject comparisons respectively provided by the Statistics toolbox in Matlab 2016b. For all ANOVA tests revealing significant differences, we performed Bonferronicorrected post-hoc tests for further insights. To investigate the relationship between motion and amygdala regulation capability, we calculated Spearman correlations between MFD per run with the extracted amygdala \% signal change (see 2.5.5) from GLM NO NUISANCE during the respective run using SPSS (IBM SPSS Statistics 25). Calculations of effect sizes, Cohen's d, have been performed for ANOVAs with multiple groups, based on group means (Jacob Cohen, 1988, p. 273) or for within group comparisons by subtracting the means and dividing 232 by the pooled standard deviations. 
233 HCP data validation: To assess group differences in MFD, HR, HRV and RVT within the HCP data between the 234 different tasks, we performed 2-tailed independent sample t-tests on the averaged values from each participant per 235 task using the Matlab 2011b Statistics Toolbox.

Interaction of amygdala regulation task with motion and physiological confounds: To compare within-

subjects' differences in amygdala BOLD activity between the three GLMs we transferred the amygdala data for each participant and run into R (R-project R3.4.0). Specifically, we applied a mixed effect model using REML with random slopes and intercepts for each run and each type of GLM. We modelled the main effects of GLM type, run, and their interaction as within-subject factors. These analyses allowed us to investigate whether estimated learning effects from real-time fMRI NF reflect motion and physiological confounds. In addition, we the analyses allowed us to investigate whether statistical group differences in NF learning are affected by noise correction.

\section{Results}

\subsection{Motion differences (How does motion vary during the neurofeedback experiment?)}

247 To assess differences in head motion we calculated MFD values for all groups from both amygdala NF 248 neurofeedback studies. In detail, we compared following aspects.

249 3.1.1 Overall group comparisons (Does motion vary as a function of intervention group?)

250 Our two independent studies revealed differences in median displacement values for head motion between 251 feedback and control groups (3T: ANOVA F $(4,8)=10.9, \mathrm{p}=.002$, Cohen's d=4.2; 7T: $\operatorname{ANOVA~F}(4,4)=16.24, \mathrm{p}=.01$, 252 Cohen's d=6.8). Bonferroni corrected post-hoc t-tests revealed higher motion in all NF groups compared to CON

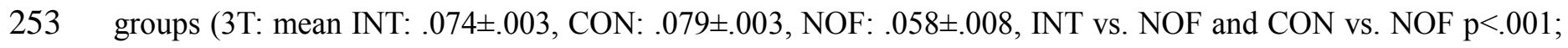

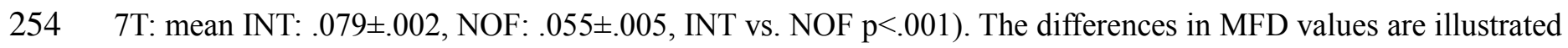
255 along the columns in Figure 1 where the black lines indicate the group averages.

256 Given that the central moment tested above revealed differences between the groups, we additionally analyzed 257 non-parametric distribution parameters, namely skewness and kurtosis, between the groups. For both parameters 
and studies, analysis revealed no differences in these distribution parameters (3T: all ANOVA F $(4,14)<1.04, \mathrm{p}>$.4;

260 The mean values of the MDF values do vary according to the intervention groups with higher motion in all NF

261 groups.
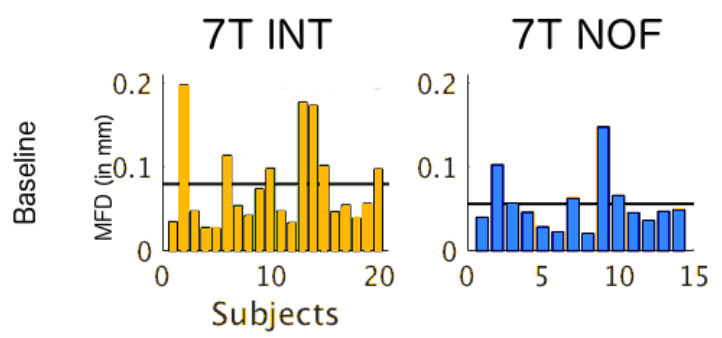

3T INT
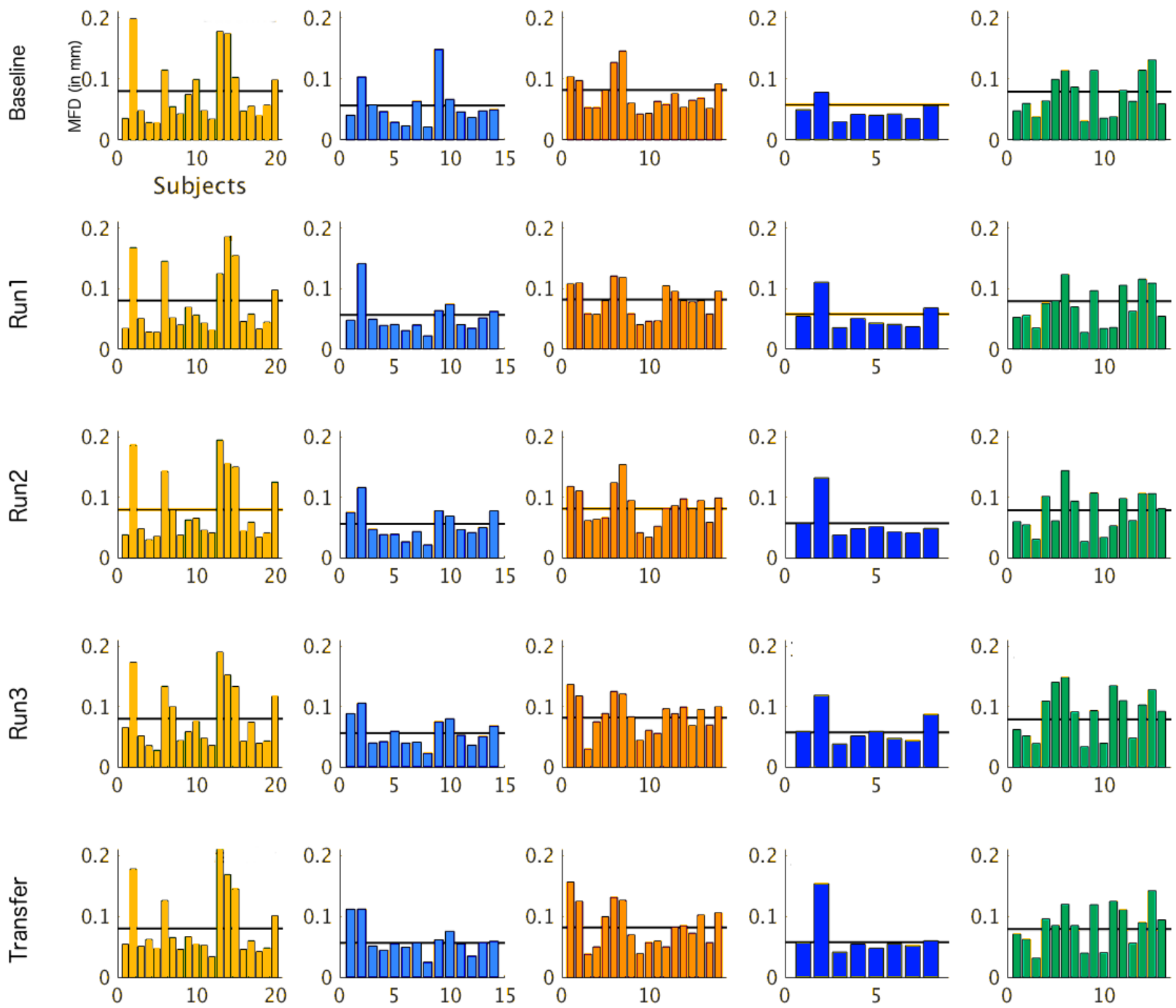

Fig. 2 Median frame-wise displacement (MFD) in mm is plotted for every subject for (A) 7T intermittent feedback, (B) 7T no feedback, (C) $3 T$ intermittent feedback, (D) $3 T$ no feedback, and (E) $3 T$ control group. The differences along the runs of the NF paradigm are plotted along the rows. The black horizontal line indicates the group average of MFD values within the respective group. 
265 3.1.2 Condition-wise comparisons (Does motion vary as a function of regulation condition?)

266 For both studies and within each group, we tested whether the MDF values vary according to the regulation 267 conditions. ANOVA testing revealed differences in head motion between HAPPY, COUNT and REST. The post268 hoc t-tests have shown in all groups increased head motion during the REST condition. For readability, we 269 summarized all statistical values in Table 1 presenting the mean values, standard deviations for each group and 270 condition combined with ANOVA F- and p-value. Additionally, the directionality of the differences and their effect 271 size are summarized in the last column. The MDF values vary as function of the regulation condition in all groups 272 of both studies regardless of the NF intervention.

\begin{tabular}{|c|c|c|c|c|c|c|c|c|c|}
\hline \multirow{2}{*}{$\begin{array}{c}\text { Condition/ } \\
\text { group }\end{array}$} & \multicolumn{2}{|c|}{ HAPPY } & \multicolumn{2}{|c|}{ COUNT } & \multicolumn{2}{|c|}{ REST } & \multirow[b]{2}{*}{$\mathrm{F}(2,8)$} & \multirow[b]{2}{*}{$\mathrm{p}=$} & \multirow[b]{2}{*}{ post-hoc p \& Cohen's d } \\
\hline & mean & std & mean & std & mean & std & & & \\
\hline \multirow[t]{2}{*}{ 7T INT } & .085 & .002 & .078 & .004 & .083 & .007 & 6.93 & .02 & HAPPY vs COUNT $p=.002, d=2.2$ \\
\hline & & & & & & & & & REST vs. COUNT $\mathrm{p}=.004, \mathrm{~d}=.87$ \\
\hline \multirow[t]{2}{*}{$7 \mathrm{~T}$ NOF } & .057 & .003 & .057 & .004 & .063 & .003 & 17.4 & .001 & REST vs. HAPPY $\mathrm{p}=.002, \mathrm{~d}=2$ \\
\hline & & & & & & & & & REST vs. COUNT $p=.004, d=1.7$ \\
\hline 3T INT & .076 & .004 & .083 & .01 & .092 & .004 & 15.2 & .002 & REST vs. HAPPY $\mathrm{p}=.002, \mathrm{~d}=4$ \\
\hline $3 \mathrm{~T}$ NOF & .054 & .007 & .059 & .007 & .067 & .013 & 9.46 & .008 & REST vs. HAPPY $\mathrm{p}=.007, \mathrm{~d}=1.2$ \\
\hline \multirow[t]{2}{*}{$3 T \mathrm{CON}$} & .078 & .001 & .077 & .007 & .087 & .008 & 12.3 & .004 & REST vs. HAPPY $\mathrm{p}=.01, \mathrm{~d}=0.99$ \\
\hline & & & & & & & & & REST vs. COUNT $p=.006, d=1.3$ \\
\hline
\end{tabular}

Table 1: ANOVA statistics with $F$ and p values and post-hoc t-test results showing directions of differences in head motion within the groups. All our groups revealed significant differences between the regulation conditions while head motion is higher during REST blocks in all groups (condition with increased mean always named first).

\section{$273 \quad 3.1 .3$ Dynamics of motion (Does motion vary over time?)}

274 To test whether within the subjects the head motion is changing over time, we tested within each group for 275 individual differences along the five runs. Notably, for all groups there are no changes in MDF values within the

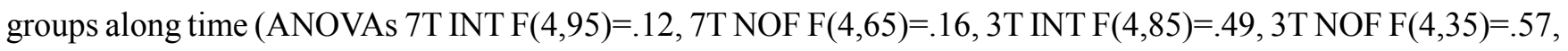


278 each participant along the rows of Figure 1 in each group. We found no significant changes in head motion over 279 time within participants.

281 We calculated the Spearman correlations between the up-regulation success of the amygdala BOLD signal with 282 the median framewise displacement values according to the groups collapsed across both studies (INT Baseline: $283 \mathrm{r}_{\mathrm{s}}=-.24, \mathrm{p}=.15 ;$ run $1: \mathrm{r}_{\mathrm{s}}=-.1, \mathrm{p}=.54 ;$ run2: $\mathrm{r}_{\mathrm{s}}=-.37, \mathrm{p}=.02 ;$ run $3: \mathrm{r}_{\mathrm{s}}=-.19, \mathrm{p}=.26$; transfer: $\mathrm{r}_{\mathrm{s}}=.13, \mathrm{p}=.46$; NOF Baseline: $284 \mathrm{r}_{\mathrm{s}}=-.24, \mathrm{p}=.3 ;$ run1: $\mathrm{r}_{\mathrm{s}}=-.17, \mathrm{p}=.45 ;$ run2: $\mathrm{r}_{\mathrm{s}}=-.21, \mathrm{p}=.35 ;$ run3: $\mathrm{r}_{\mathrm{s}}=-.27, \mathrm{p}=.25 ;$ transfer: $\left.\mathrm{r}_{\mathrm{s}}=-.15, \mathrm{p}=.51\right)$. Taking 285 Bonferroni corrections for multiple comparisons into account, Spearman correlations between the up-regulation 286 success and the amount of head motion did not reach significance.

287

\subsection{Physiological data differences (How do physiological parameters vary during the neurofeedback experiment?)}

Comparable to the median framewise displacements, we analyzed differences in the physiological parameters of HR, HRV and RVT using ANOVA testing within and between groups.

\subsubsection{Overall group comparisons (Do they vary as a function of intervention group?)}

Our ANOVA analysis comparing physiological parameters between groups revealed a significantly higher heart

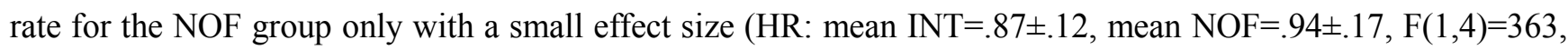
$\mathrm{p}<.001$, Cohen's d=.49). HRV and RVT did not show differences between the groups (HRV mean INT=.0627 \pm .03 , mean $\mathrm{NOF}=.0632 \pm .032, \mathrm{~F}(1,4)=.02, \mathrm{p}=.86$, Cohen's $\mathrm{d}=.02$; RVT mean $\mathrm{INT}=.44 \pm .15$, mean $\mathrm{NOF}=.48 \pm .16$, $\mathrm{F}(1,4)=5.9, \mathrm{p}=.07$, Cohen's $\mathrm{d}=.26$ ). The distribution of all parameters within both groups are shown as violin plots in Figure 4 with HR (Fig 4A), HRV (Fig. 4B) and RVT (Fig. 4C), each compared to HCP data (see 3.3)

\subsubsection{Condition-wise comparisons (Do they vary as a function of regulation condition?)}

Our analysis revealed differences in HR, HRV and RVT between the conditions HAPPY, COUNT and REST periods in both INT and NOF group independently. Table 2 summarizes all average values and standard deviation together with ANOVA statistics and post-hoc t-tests. RVT values show lower values during COUNT condition in both groups, while HR is increased during COUNT compared to both other conditions. Further 


\begin{tabular}{|c|c|c|c|c|c|c|c|c|c|}
\hline \multirow{3}{*}{$\begin{array}{c}\text { Condition/ } \\
\text { group }\end{array}$} & \multicolumn{4}{|c|}{ COUNT } & \multicolumn{2}{|c|}{ REST } & & & \\
\hline & \multicolumn{9}{|c|}{ Heart Rate (HR) } \\
\hline & mean & std & mean & Std & mean & std & $\mathrm{F}(2,8)$ & $\mathrm{p}=$ & post-hoc p \& Cohen's d \\
\hline \multirow[t]{3}{*}{$7 \mathrm{~T}$ NOF } & .93 & .16 & .95 & .16 & .94 & .17 & 46.4 & .001 & COUNT vs. HAPPY $\mathrm{p}<.001, \mathrm{~d}=.12$ \\
\hline & & & & & & & & & COUNT vs. REST $\mathrm{p}<.001, \mathrm{~d}=.06$ \\
\hline & \multicolumn{9}{|c|}{ Heart rate variability (HRV) } \\
\hline \multirow{2}{*}{$7 \mathrm{~T}$ NOF } & & & & & & & & & HAPPY vs. COUNT $\mathrm{p}=.003, \mathrm{~d}=$ \\
\hline & \multicolumn{9}{|c|}{ Respiratory volume per time unit (RVT) } \\
\hline \multirow[t]{2}{*}{ 7T INT } & .44 & .15 & .43 & .15 & .46 & .16 & 15.2 & .002 & HAPPY vs COUNT $\mathrm{p}=.05, \mathrm{~d}=$ \\
\hline & & & & & & & & & REST vs. COUNT $p=.002, d=$. \\
\hline \multirow[t]{2}{*}{$7 \mathrm{~T}$ NOF } & .48 & .16 & .45 & .15 & .5 & .16 & 44.5 & $<.001$ & HAPPY vs. COUNT $\mathrm{p}<.001, \mathrm{~d}=$ \\
\hline & & & & & & & & & REST vs. COUNT $\mathrm{p}<.001, \mathrm{~d}=$ \\
\hline
\end{tabular}

$303 \quad 3.2 .3$ Dynamics of motion (Do they vary over time?)

304 To test whether within the subjects HR, HRV or RVT are changing over time, we tested within each group for 305 individual differences along the five runs. Notably, for all groups and parameters we found no changes within the 306 groups along time (all 7T INT F(4,93)<.87, $\mathrm{p}>.48$, 7T NOF all $\mathrm{F}(4,65)<.99, \mathrm{p}>.47$, all Cohen's $\mathrm{d}<.3$ ). The 307 physiological parameters do not vary over time.

308 3.2.4 Correlation Regulation capability and head motion (Do they correlate with learning effects?)

309 We calculated the Spearman correlations between the up-regulation capability with HR, HRV and respiration 310 values (see summary in Table 2) for INT and NOF group along all runs.

$\begin{array}{rrrrrr}\text { Baseline } & \text { Run1 } & \text { Run2 } & \text { Run3 } & \text { Transfer } & \\ \mathrm{r}_{\mathrm{s}} & \mathrm{r}_{\mathrm{s}} & \mathrm{r}_{\mathrm{s}} & \mathrm{r}_{\mathrm{s}} & \mathrm{r}_{\mathrm{s}} & \text { each } \mathrm{p}>\end{array}$




\begin{tabular}{lcccccc}
\multicolumn{7}{c}{ Heart rate } \\
7T INT & .36 & .14 & -.05 & .04 & .08 & .14 \\
7T NOF & .2 & .31 & .45 & -.24 & -.005 & .12 \\
& & & Heart rate variability & & \\
7T INT & -.39 & .31 & -.17 & .03 & .07 & .11 \\
7T NOF & .26 & .58 & .035 & .45 & -.54 & .04 \\
& & & Respiratory volume & & .02 \\
7T INT & .06 & .13 & .04 & -.19 & -.56 & .05 \\
7T NOF & -.44 & .55 & .37 & .2 & .15 & \\
$*$ significance level $\mathrm{p}<.01$ with Bonferroni-correction for multiple comparisons &
\end{tabular}

Table 3: Correlation values between amygdala extracted regulation success in BOLD \% signal changes and physiological noise variables along all runs.

311 Comparable to the median framewise displacements, we revealed no correlations between amygdala regulation 312 success from BOLD \% signal with any of the physiological parameters that might explain the NF training effects 313 in the INT group.

\subsection{Validation analysis HCP data}

315 In order to assess whether our findings for MFD and physiological parameters fall within standard parameter 316 variability we examined data from HCP. Furthermore, we used these data to examine whether parameter changes 317 revealed from our data correspond to parameter changes observed in different tasks in HCP data, where these tasks 318 differed in levels of required mental engagement. Therefore, we used a big sample of HCP data comprising REST1, 319 EMOTION, and MOTOR tasks. We extracted and analyzed MFD values and physiological parameters from the 320 HCP sample data for each task analogue to our data sets. Overall, all parameter values we obtained for our small 321 sample size are within the distribution of parameter ranges observed from the big HCP dataset. With regard to 322 MFD values, we found significantly higher MFD values in MOTOR vs. REST1 (difference in MFD=.007 mm, $323 \mathrm{p}<.001$ ) and EMOTION vs. REST1 (difference in MFD=.011, $\mathrm{p}<.001$ ). The higher head motion between 324 EMOTION and REST1 data from HCP mirrors our findings between feedback groups (INT, CON) and control 325 groups (NOF), although the NOF groups performed the mental tasks of happy memories and counting backwards 326 but without any visual feedback. Figure 3 summarizes the MFD values for all three samples (7T, 3T and HCP) 
averaged across all runs within each participant (Note that MOTOR task is not illustrated since results are similar to EMOTION).

329 With regard to physiological parameters, HCP data revealed significantly higher HR values in both EMOTION 330 and MOTOR compared to REST1 (difference EMOTION vs. REST1 = $2.2 \mathrm{bpm}$, difference MOTOR vs. REST1 $331=1.5 \mathrm{bpm}, \mathrm{p}<.001)$. Furthermore, we found lower HRV in EMOTION compared to REST (difference=-.017) but 332 higher HRV in MOTOR vs. REST (difference $=.007, \mathrm{p}<.001$ ). For RVT, both tasks revealed significantly higher 333 values in both tasks compared to REST1 (difference $=.16 ;=.07 ; \mathrm{p}<.001$ ). Figure 4 summarizes heart rate (A), HRV

334 (B) and RVT (C) values from our 7T dataset and HCP sample averaged across all runs within each subject.
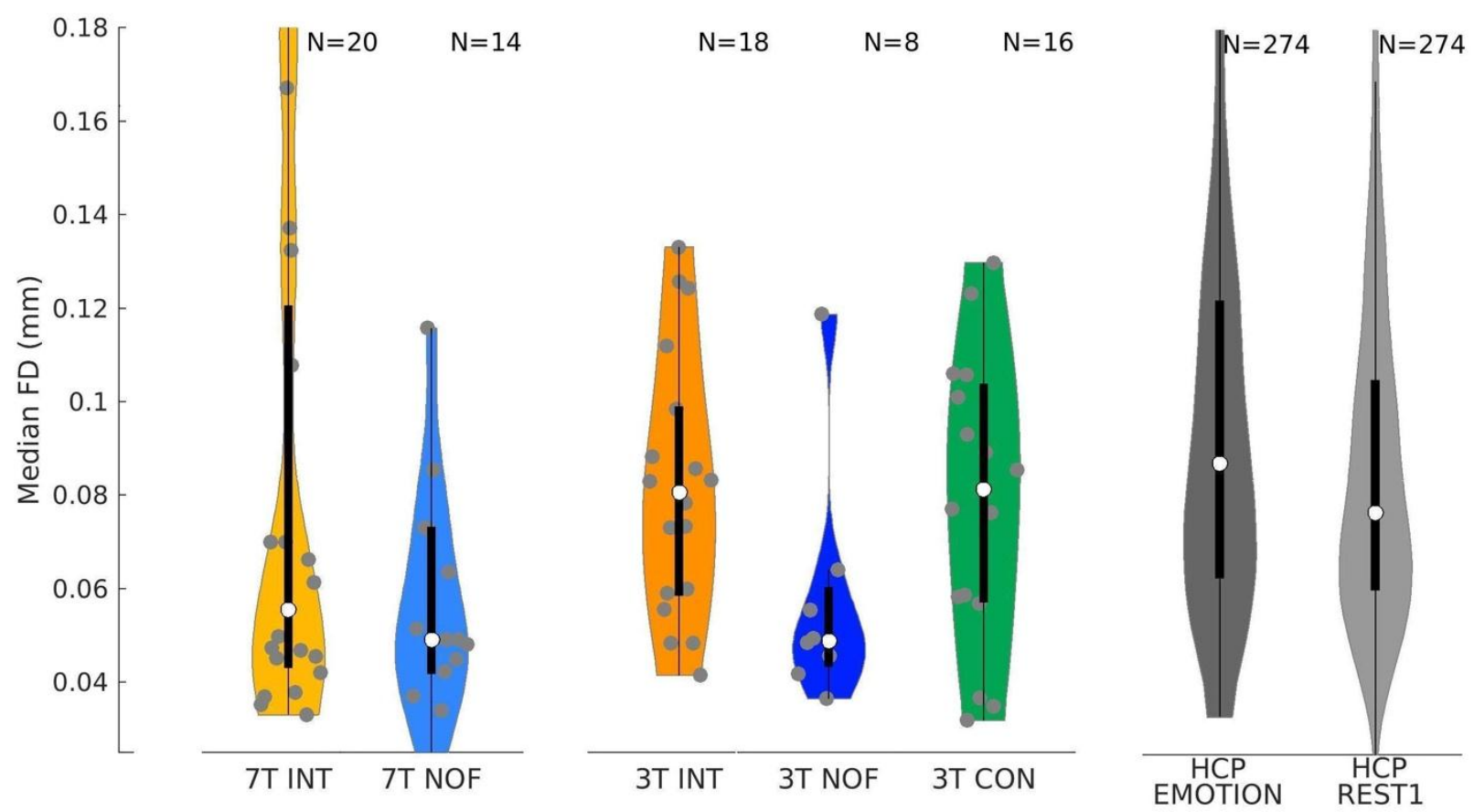

Fig. 3 Comparison of movement in our data with the HCP data. The MFD parameter ranges we observed in our data sets fall within the normal range of parameter values. Moreover, task-based fMRI resulted in higher MFD values than resting-state fMRI in the HCP data. This finding validates our findings from both rt-fMRI amygdala NF data sets with a bigger sample size. 
bioRxiv preprint doi: https://doi.org/10.1101/366138; this version posted July 13, 2018. The copyright holder for this preprint (which was not certified by peer review) is the author/funder, who has granted bioRxiv a license to display the preprint in perpetuity. It is made available under aCC-BY-NC-ND 4.0 International license.

A

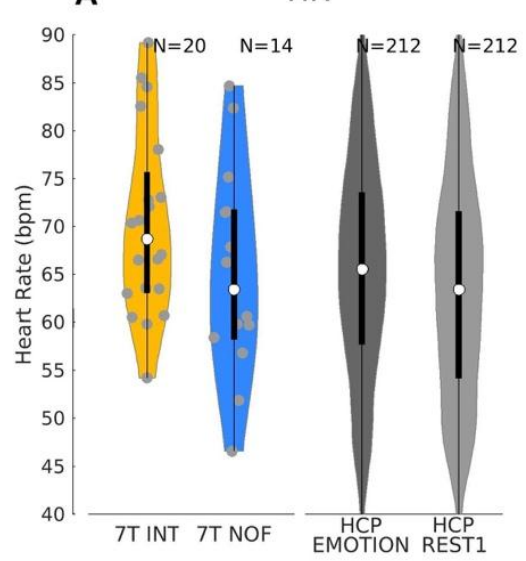

B

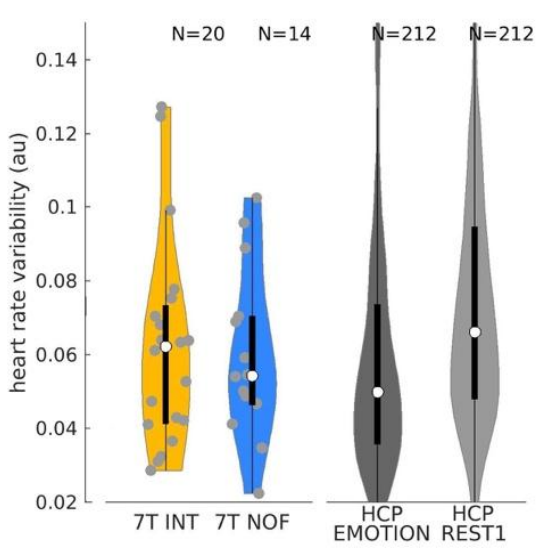

C

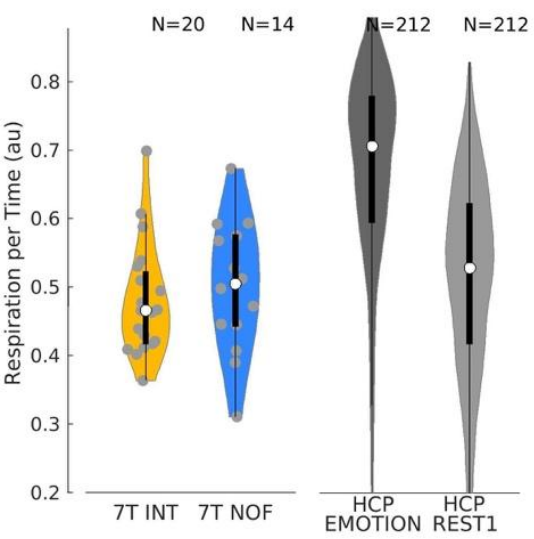

Fig. 4 Comparison of physiological parameters in our data with the HCP data. To validate our findings for heart rate, heart rate variability and respiration volume, we analyzed the emotional task and resting state data from the HCP project. The parameter ranges in our data were comparable to those of the HCP sample. Second, although our sample did reveal significant differences in HR only with a small effect size, the bigger sample from the HCP data revealed significant differences in heart rate (A), heart rate variability $(B)$ and respiration volume (C). 
3.4 Amygdala task-motion and task-physiological interactions (How does the main outcome of an rt-fMRI study potentially change when motion and physiological parameters have been accounted for?)

\section{$341 \quad 3.4 .1 \quad$ Exemplary single participant comparison}

342 Exemplarily, we show a representative participant from the 7T INT group comparing amygdala \% signal change 343 extractions from the three GLMs including different numbers of nuisance regressors from no nuisance regressors 344 over motion regressors only to motion combined with physiological regressors - GLM NO NUISANCE (Fig. 5 left), GLM + MOCO (Fig. 5 middle), and GLM + MOCO \& PHYSIO (Fig. 5 right). For this participant, the comparison between GLM NO NUISANCE and GLM + MOCO \& PHYSIO revealed no differences between the extracted values $(p=.46)$. We inspected these values from all our participants' data observing changes in the

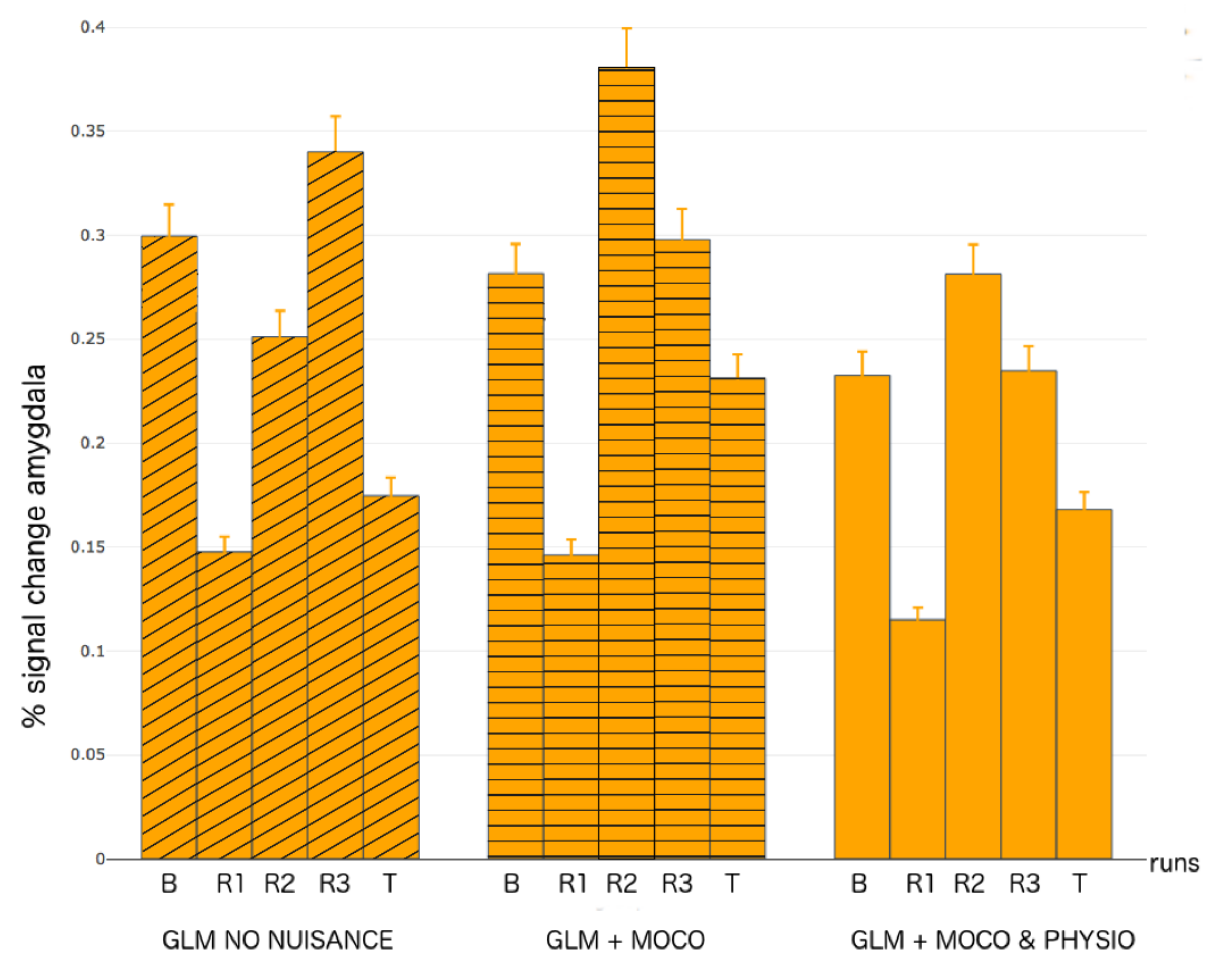

Fig. 5 Single participant comparison of learning effects: Comparison of GLMs comprising no nuisance regression (GLM NO NUISANCE), motion regression only (GLM MOCO), and motion and physiological noise regression (GLM MOCO \& PHYSIO) for one participant of 7T INT group. We found no differences regarding the percentage signal change extraction values between the GLM NO NUISANCE and GLM + MOCO \& PHYSIO. 


\section{$351 \quad 3.4 .2$ Group level comparison 7T and 3T}

352 For group-level comparisons, we compared the \% signal change in amygdala along the runs between the three different GLMs - GLM NO NUISANCE, GLM + MOCO, and GLM + MOCO \& PHYSIO - using linear mixed $\mathrm{p}=.02)$. For both $3 \mathrm{~T}$ groups we found no main effects on GLM-type $\left(\chi^{2}(1)=3.1, \mathrm{p}=.08 ; \chi^{2}(1)=2.8, \mathrm{p}=.1\right)$. Figures 6 , types along the five runs. The cyan diamonds indicate the average values. We found

\subsubsection{Effects of GLM enhancement on NF group differences}

360 To answer how far statistical group differences in NF learning will be affected by such an enhanced noise 361 correction, we performed mixed effect model analysis using INT and NOF group as between-subject factor and 362 run as within-subject-factor. Critically, we found a difference in the resulting overall learning effects between the 363 INT and NOF group for the comparison of amygdala signal changes. The learning effects based on the GLM NO 364 NUISANCE would lead to significant main effects of group $\left(\chi^{2}(1)=3.9, p=.047\right)$ and run $\left(\chi^{2}(3)=9.6, p=.021\right)$ but 365 no interaction. Repeating this test with the GLM + MOCO \& PHYSIO evolves strong trends towards these results 366 (main effect for group $\chi^{2}(1)=2.8, p=.09$ and run $\chi^{2}(3)=4.3, p=.22$ ) and also no interaction. Our results show that 367 the additional noise regressors on first level decreases the statistical significance of the results although the interpretation remains. 
bioRxiv preprint doi: https://doi org/10.1101/366138; this version posted July 13,2018 . The copyright holder for this preprint (which was not

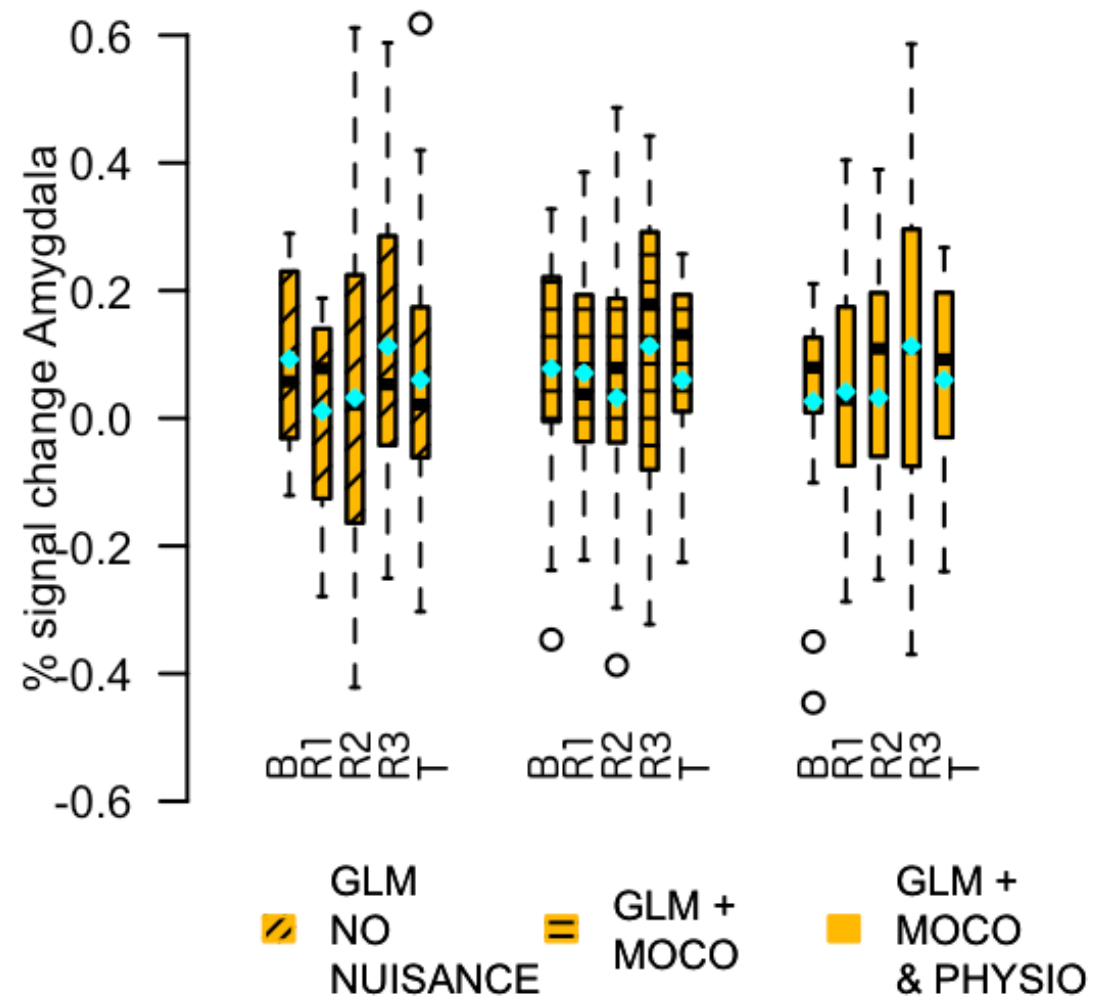

Fig. 7 7T INT group comparison: Comparison of amygdala extracted \% signal change from GLMs without nuisance regressors, motion regressors only and motion plus physiological noise regressors. The additional noise regressors did not change the results significantly.

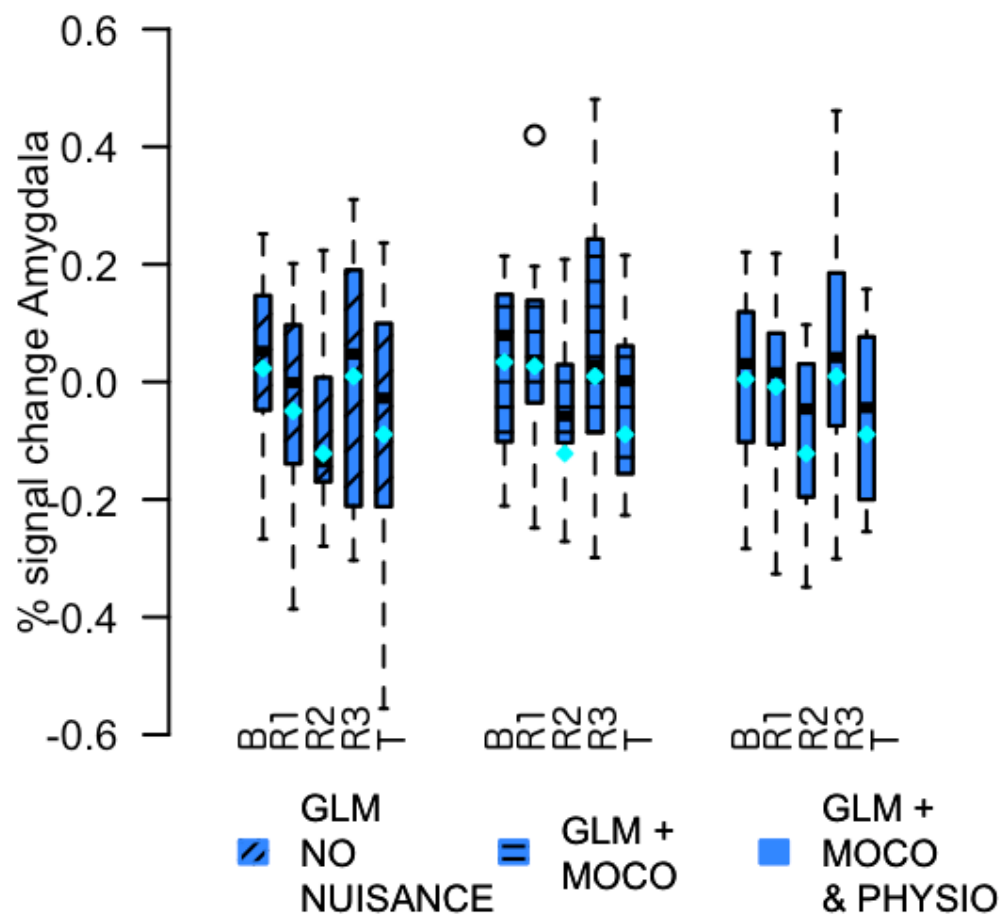

Fig. 67 T NOF group comparison: Comparison of amygdala extracted \% signal change from GLMs without nuisance regressors, motion regressors only and motion plus physiological noise regressors. The additional regression of noise reveals a main effect of GLM comparison within this control group. 
3T INT

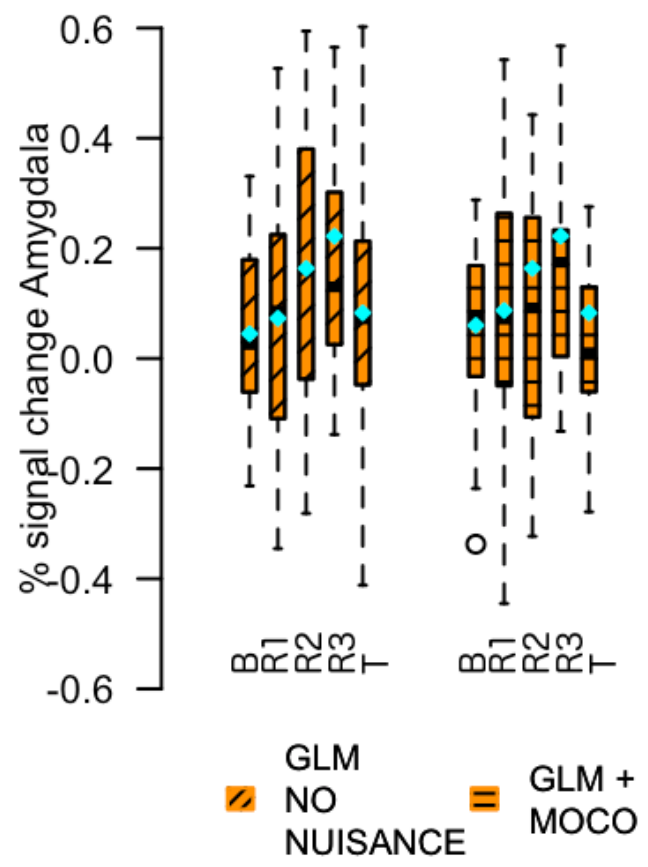

3T NOF

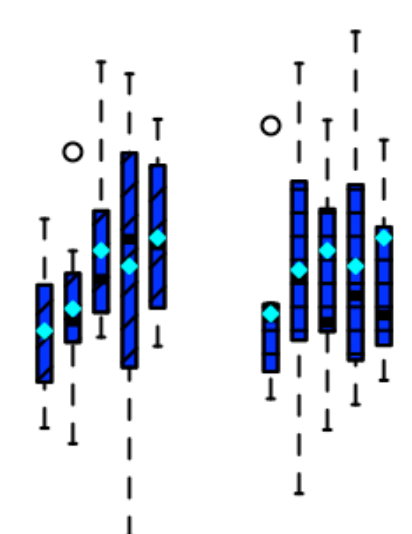

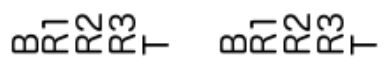

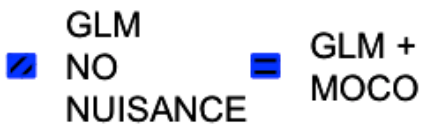

Fig. 83 T INT and NOF group comparison: Comparison of amygdala extracted \% signal change from GLMs without nuisance regressors and motion regressors. The additional regression of motion did not change the results significantly.

\section{Discussion}

373 In two independent amygdala neurofeedback studies, we systematically investigated effects of head motion, heart 374 rate, heart rate variability and respiratory volume on BOLD signal changes achieved from neurofeedback training.

375 We mainly asked three questions: (1) How do these parameters vary during a neurofeedback experiment and if 376 they vary as a function of either intervention (feedback or no feedback), or regulation condition (HAPPY, COUNT, 377 REST), or along training runs (Baseline, Run1, Run2, Run3, Transfer)? In response to this, our results revealed 378 that motion differed between groups and between regulation condition. For physiological parameters, we found a 379 slightly higher heart rate in the no feedback group with a small effect size. Furthermore, the parameters differ 380 between regulation conditions inconsistently with very small effect sizes. All nuisance parameters did not vary along training runs. (2) Do these parameters correlate with learning effects, i.e. increase together with increased capacity of BOLD regulation across several training runs? Here, our results have shown that all nuisance 
3.2.4). (3) How does the main outcome of an rt-fMRI study (i.e. the demonstration of BOLD regulation and its transfer) potentially change when motion and physiological parameters have been accounted for? In response to this question, we found that including all nuisance regressors properly did not change the interpretation of the results but affected the statistical results in terms of a decrease in statistical power (see 3.4.3). Therefore, we conclude that overall changes in BOLD activity achieved from amygdala neurofeedback training are not mainly driven by noise. Our results underline that neurofeedback allows to learn self-regulation of brain activity reliably with ongoing training.

Our analysis of the HCP data showed that as engagement with the task or the presented stimuli increases, by going from a resting state (i.e. REST1) task to a more active task (i.e. EMOTION or MOTOR), motion and physiological parameters change significantly. In particular, motion increases, heart rate and respiration increase, whereas heart rate variability decreases. This is in line with reported literature describing the effects of these parameters on BOLD (Lund et al., 2005; Sakaki et al., 2016; Thayer, 2018). Furthermore, it has been shown that higher heart rate variability in self-control is associated with altered brain activity in ventromedial prefrontal cortex but not amygdala (Maier and Hare, 2017). The results in our studies confirm to these findings, especially as we found in both groups only within-subject differences between the regulation conditions.

The combined evidence indicates that the reason behind the observed differences between our groups might be the feedback information, which might lead to a stronger engagement of the participants in the task. The presence of the feedback and its interpretation adds a cognitive process for the participants, since the level of the stimulus needs to be evaluated/consolidated with the own current belief of their performance. Furthermore, this allows to evaluate whether the strategy employed before the feedback aroused needs to be changed. This entails the process of learning over BOLD signal even with different kinds of feedback - either presented continuously or occasionally only every 40 seconds. Critically to our study design, we used only one control group instructed identical with regard to strategies but receiving no feedback at all. But with regard to behavioral differences, we found no differences in strategy usage or willingness to perform the task between our groups. The compliance of the control groups is also underpinned by our findings from HCP analysis revealing significant differences between restingstate and task-fMRI, while our groups do not differ significantly in physiological parameters. 
410 One could argue that the amygdala BOLD effects may be mainly driven by these physiological factors, especially 411 considering the publication of Boubela et al. (2015) warning exactly for these possible effects. It therefore is 412 especially essential to control for these confounders. Although in our results this is clearly not the case as the 413 amygdala BOLD variability across participants is not correlated with any motion or physiological parameters, 414 potential confounds of head motion and physiological parameters needs to be taken into account, especially when 415 analyzing average extracted amygdala BOLD effects. When they have been extracted with a GLM approach that includes motion and physiology, the degrees of freedom at first level are decreased due to the additional motion $(\mathrm{N}=12)$ and physiological $(\mathrm{N}=8)$ regressors, which could lead to a decreased statistical power when assessing group differences (Faul et al., 2009). Therefore, an appropriate sample size is needed.

Regarding motion, there is a conflicting report showing decreased motion going from resting-state to task-based fMRI (Huijbers et al., 2017), which conflicts with findings from our own studies (3T and 7T) and also (more clearly) conflicts with our findings from the analysis of 822 participants from the HCP. However, Huijbers at al. state the possible effects of different kinds of cognitive tasks they used, use mainly clinical population from different psychiatric disorders and age effects on their reported motion values. The HCP data comprise a lower age range with overall less motion and comprises healthy adult participants.

Limitations: A critical aspect to our study might be that the different physiological and motion signals we assess come with different time resolutions. Due to the nature of fMRI the correction methods we have used are within the repetition time of our sequence (2s). Aside, the data we acquired are in high resolution (1.4 mm isotropic) at ultra-highfield strength, which has been shown to be more sensitive to physiological noise than standard $3 \mathrm{~T}$ sequences, although different parts of the brain are affected differently by physiological changes (van der Zwaag et al., 2015). However, we used the current state-of-the-art methods for data acquisition and nuisance correction. 431 Overall, our results cannot be fully generalized to all kinds of rt-fMRI neurofeedback studies and it is crucial that more fMRI-NF studies report noise corrected results.

Although our study underpins the reliability of neurofeedback allowing to increase BOLD activity over the training, we think it is highly recommended to acquire motion and physiological data, and also highlights the importance of post-hoc offline analysis taking these into account when analyzing the data. This would help to increase trust in 
all studies reporting neurofeedback training effects. However, our results indicate that there is not crucial alteration of the main conclusions of the experiment, and therefore, no pressing need for the rt-fMRI field to invest in the implementation of a real-time motion and physiological correction for the on-line extraction of BOLD activity that is used as the neurofeedback in the experiment. At the moment, this would be only possible if an EEG system is used that supports real-time data export (for example Brain Products Recorder or EGI Netstation). EEG systems are not always available and would increase the complexity of the setup unnecessarily. Second, a real-time analysis they would be able to be used as regressors for on-line statistics.

444 Nevertheless, our recommendation for further studies is to carefully report in addition to study results a scheme of 445 neurofeedback learning effects with post-hoc regression of physiological noise parameters to prove the validity of 446 the changes in neural activity.

\section{Conclusions}

449 We conclude that motion artifacts did not drive the improvement in self-regulation capability of the amygdala and other feedback related brain regions. But the differences in motion parameters between feedback and control groups emphasize the importance to control for such confounders. Furthermore, we strongly suggest to monitor 


\section{Acknowledgements}

459 The authors would like to thank Renate Blobel for all the help in recruitment and scanning at the 7T

460 MR scanner. We also thank Maria Watermann and Maya Nathan for help with recruitment. We also

461 thank Myung-Ho In from the Department of Biomedical Magnetic Resonance, Magdeburg, for getting

462 started with the MR sequence. Furthermore, we thank Saskia Bollmann for personal help with the

463 usage of TapasIO Toolbox. This work was supported by grant 00014_165884 from the Swiss National

464 Science Foundation (to PNT) and from the European Union's Horizon 2020 research and innovation

465 programme under the Marie Sklodowska-Curie grant agreement 794395 (to LH).

466 The authors declare no competing financial interests. 


\section{References}

Ashburner, J., 2007. A fast diffeomorphic image registration algorithm. Neuroimage 38, 95-113. https://doi.org/http://dx.doi.org/10.1016/j.neuroimage.2007.07.007

Birn, R.M., Diamond, J.B., Smith, M.A., Bandettini, P.A., 2006. Separating respiratory-variationrelated fluctuations from neuronal-activity-related fluctuations in $\{\mathrm{fMRI}\}$. Neuroimage 31, 15361548. https://doi.org/10.1016/j.neuroimage.2006.02.048

Boubela, R.N., Kalcher, K., Huf, W., Seidel, E.-M., Derntl, B., Pezawas, L., Našel, C., Moser, E., 2015. fMRI measurements of amygdala activation are confounded by stimulus correlated signal fluctuation in nearby veins draining distant brain regions. Sci. Rep. 5, 10499. https://doi.org/10.1038/srep10499

Brühl, A.B., Scherpiet, S., Sulzer, J., Stämpfli, P., Seifritz, E., Herwig, U., 2014. Real-time neurofeedback using functional MRI could improve down-regulation of amygdala activity during emotional stimulation: A proof-of-concept study. Brain Topogr. 27, 138-148. https://doi.org/10.1007/s10548-013-0331-9

Chang, C., Cunningham, J.P., Glover, G.H., 2009. Influence of heart rate on the BOLD signal: The cardiac response function. Neuroimage 44, 857-869. https://doi.org/10.1016/j.neuroimage.2008.09.029

Chao-Gan, Y., Yu-Feng, Z., 2010a. DPARSF: A MATLAB Toolbox for “ Pipeline " Data Analysis of Resting - State fMRI. Front. Syst. Neurosci. 4, 13. https://doi.org/10.3389/fnsys.2010.00013

Chao-Gan, Y., Yu-Feng, Z., 2010b. DPARSF: A MATLAB Toolbox for " Pipeline " Data Analysis of Resting - State fMRI. Front. Syst. Neurosci. 4, 13. https://doi.org/10.3389/fnsys.2010.00013

Deichmann, R., Josephs, O., Hutton, C., Corfield, D.R., Turner, R., 2002. Compensation of Susceptibility-Induced BOLD Sensitivity Losses in Echo-Planar fMRI Imaging. Neuroimage 15, 120-135. https://doi.org/10.1006/nimg.2001.0985

Faul, F., Erdfelder, E., Buchner, A., Lang, A.-G., 2009. Statistical power analyses using G*Power 3.1: Tests for correlation and regression analyses. Behav. Res. Methods 41, 1149-1160. https://doi.org/10.3758/BRM.41.4.1149 
Gerin, M.I., Fichtenholtz, H., Roy, A., Walsh, C.J., Krystal, J.H., Southwick, S., Hampson, M., 2016. Real-Time fMRI Neurofeedback with War Veterans with Chronic PTSD: A Feasibility Study. Front. Psychiatry 7, 111. https://doi.org/10.3389/fpsyt.2016.00111

Goebel, R., 2012. BrainVoyager — Past, present, future. Neuroimage 62, 748-756. https://doi.org/10.1016/J.NEUROIMAGE.2012.01.083

Goscinski, W.J., McIntosh, P., Felzmann, U.C., Maksimenko, A., Hall, C.J., Gureyev, T., Thompson, D., Janke, A., Galloway, G., Killeen, N.E.B., Raniga, P., Kaluza, O., Ng, A., Poudel, G., Barnes, D., Nguyen, T., Bonnington, P., Egan, G.F., 2014. The multi-modal Australian ScienceS Imaging and Visualization Environment (MASSIVE) high performance computing infrastructure: applications in neuroscience and neuroinformatics research. Front. Neuroinform. 8. https://doi.org/10.3389/fninf.2014.00030

Hellrung, L., Dietrich, A., Hollmann, M., Pleger, B., Kalberlah, C., Roggenhofer, E., Villringer, A., Horstmann, A., 2018. Intermittent compared to continuous real-time fMRI neurofeedback boosts control over amygdala activation. Neuroimage 166. https://doi.org/10.1016/j.neuroimage.2017.10.031

Hellrung, L., Hollmann, M., Zscheyge, O., Schlumm, T., Kalberlah, C., Roggenhofer, E., Okon-Singer, H., Villringer, A., Horstmann, A., 2015. Flexible adaptive paradigms for fMRI using a novel software package 'brain analysis in real-time' (BART). PLoS One 10. https://doi.org/10.1371/journal.pone.0118890

Hinds, O., Ghosh, S., Thompson, T.W., Yoo, J.J., Whitfield-Gabrieli, S., Triantafyllou, C., Gabrieli, J.D.E., 2011. Computing moment-to-moment BOLD activation for real-time neurofeedback. Neuroimage 54, 361-368. https://doi.org/10.1016/j.neuroimage.2010.07.060

Hollmann, M., Mönch, T., Mulla-Osman, S., Tempelmann, C., Stadler, J., Bernarding, J., 2008. A new concept of a unified parameter management, experiment control, and data analysis in fMRI: Application to real-time fMRI at $3 \mathrm{~T}$ and 7 T. J. Neurosci. Methods 175, 154-162. https://doi.org/10.1016/j.jneumeth.2008.08.013

Hollmann, M., Rieger, J.W., Baecke, S., Lützkendorf, R., Müller, C., Adolf, D., Bernarding, J., 2011. Predicting decisions in human social interactions using real-time fMRI and pattern classification. PLoS One 6, e25304. https://doi.org/10.1371/journal.pone.0025304 
motion during MRI under task than resting-state conditions. Neuroimage 147, 111-120. https://doi.org/10.1016/J.NEUROIMAGE.2016.12.002

In, M.H., Speck, O., 2012. Highly accelerated PSF-mapping for EPI distortion correction with improved fidelity. Magn. Reson. Mater. Physics, Biol. Med. 25, 183-192. https://doi.org/10.1007/s10334-011-0275-6

Jacob Cohen, 1988. Statistical Power Analysis for the Behavioral Sciences, 2nd editio. ed, Nature. Erlbaum. https://doi.org/10.1038/506274a

Jenkinson, M., Bannister, P., Brady, M., Smith, S., 2002. Improved optimization for the robust and accurate linear registration and motion correction of brain images. Neuroimage 17, 825-841. https://doi.org/10.1016/S1053-8119(02)91132-8

Jenkinson, M., Beckmann, C.F., Behrens, T.E.J., Woolrich, M.W., Smith, S.M., 2012. FSL. Neuroimage 62, 782-790. https://doi.org/10.1016/j.neuroimage.2011.09.015

Kasper, L., Bollmann, S., Diaconescu, A.O., Hutton, C., Heinzle, J., Iglesias, S., Hauser, T.U., Sebold, M., Manjaly, Z.M., Pruessmann, K.P., Stephan, K.E., 2017. The PhysIO Toolbox for Modeling Physiological Noise in fMRI Data. J. Neurosci. Methods 276, 56-72. https://doi.org/10.1016/j.jneumeth.2016.10.019

Koush, Y., Ashburner, J., Prilepin, E., Sladky, R., Zeidman, P., Bibikov, S., Scharnowski, F., Nikonorov, A., De Ville, D. Van, 2017a. OpenNFT: An open-source Python/Matlab framework for real-time fMRI neurofeedback training based on activity, connectivity and multivariate pattern analysis. Neuroimage. https://doi.org/10.1016/j.neuroimage.2017.06.039

Koush, Y., Ashburner, J., Prilepin, E., Sladky, R., Zeidman, P., Bibikov, S., Scharnowski, F., Nikonorov, A., De Ville, D. Van, 2017b. OpenNFT: An open-source Python/Matlab framework for real-time fMRI neurofeedback training based on activity, connectivity and multivariate pattern analysis. Neuroimage. https://doi.org/10.1016/j.neuroimage.2017.06.039

Lemieux, L., Salek-Haddadi, A., Lund, T.E., Laufs, H., Carmichael, D., 2007. Modelling large motion events in fMRI studies of patients with epilepsy. Magn. Reson. Imaging 25, 894-901. https://doi.org/10.1016/j.mri.2007.03.009

Lund, T.E., Nørgaard, M.D., Rostrup, E., Rowe, J.B., Paulson, O.B., 2005. Motion or activity: their role in intra- and inter-subject variation in \{fMRI $\}$. Neuroimage 26, 960-964. https://doi.org/10.1016/j.neuroimage.2005.02.021 
Maier, S.U., Hare, T.A., 2017. Higher Heart-Rate Variability Is Associated with Ventromedial Prefrontal Cortex Activity and Increased Resistance to Temptation in Dietary Self-Control Challenges. J. Neurosci. 37, 446-455. https://doi.org/10.1523/JNEUROSCI.2815-16.2016

Marxen, M., Jacob, M.J., Müller, D.K., Posse, S., Ackley, E., Hellrung, L., Riedel, P., Bender, S., Epple, R., Smolka, M.N., 2016. Amygdala Regulation Following fMRI-Neurofeedback without Instructed Strategies. Front. Hum. Neurosci. 10, 183. https://doi.org/10.3389/fnhum.2016.00183

Mugler, J.P., Brookeman, J.R., 1990. Three-dimensional magnetization-prepared rapid gradient-echo imaging (3D MP RAGE). Magn. Reson. Med. 15, 152-157. https://doi.org/10.1002/mrm.1910150117

Paret, C., Kluetsch, R., Ruf, M., Demirakca, T., Hoesterey, S., Ende, G., Schmahl, C., 2014. Downregulation of amygdala activation with real-time fMRI neurofeedback in a healthy female sample. Front. Behav. Neurosci. 8, 299. https://doi.org/10.3389/fnbeh.2014.00299

Parkes, L., Fulcher, B., Yücel, M., Fornito, A., 2018. An evaluation of the efficacy, reliability, and sensitivity of motion correction strategies for resting-state functional MRI. Neuroimage 171, 415436. https://doi.org/10.1016/j.neuroimage.2017.12.073

Sakaki, M., Yoo, H.J., Nga, L., Lee, T.-H., Thayer, J.F., Mather, M., 2016. Heart rate variability is associated with amygdala functional connectivity with MPFC across younger and older adults. Neuroimage 139, 44-52. https://doi.org/10.1016/J.NEUROIMAGE.2016.05.076

Sitaram, R., Ros, T., Stoeckel, L., Haller, S., Scharnowski, F., Lewis-Peacock, J., Weiskopf, N., Blefari, M.L., Rana, M., Oblak, E., Birbaumer, N., Sulzer, J., 2016. Closed-loop brain training: the science of neurofeedback. Nat. Rev. Neurosci. 18, 86-100. https://doi.org/10.1038/nrn.2016.164

Thayer, J.F., 2018. How heart rate variability affects emotion regulation brain networks. Curr. Opin. Behav. Sci. 19, 98-104. https://doi.org/10.1016/J.COBEHA.2017.12.017

Yan, C.-G., Cheung, B., Kelly, C., Colcombe, S., Craddock, R.C., Di Martino, A., Li, Q., Zuo, X.-N., Castellanos, F.X., Milham, M.P., 2013a. A comprehensive assessment of regional variation in the impact of head micromovements on functional connectomics. Neuroimage 76, 183-201. https://doi.org/10.1016/j.neuroimage.2013.03.004

Yan, C.-G., Cheung, B., Kelly, C., Colcombe, S., Craddock, R.C., Di Martino, A., Li, Q., Zuo, X.-N., Castellanos, F.X., Milham, M.P., 2013b. A comprehensive assessment of regional variation in the impact of head micromovements on functional connectomics. Neuroimage 76, 183-201. 
585 Young, K.D., Zotev, V., Phillips, R., Misaki, M., Yuan, H., Drevets, W.C., Bodurka, J., 2014. Real586 Time fMRI Neurofeedback Training of Amygdala Activity in Patients with Major Depressive 587 Disorder. PLoS One 9, e88785. https://doi.org/10.1371/journal.pone.0088785

588 Zotev, V., Krueger, F., Phillips, R., Alvarez, R.P., Simmons, W.K., Bellgowan, P., Drevets, W.C., 589 Bodurka, J., 2011. Self-regulation of amygdala activation using real-time FMRI neurofeedback. $590 \quad$ PLoS One 6. https://doi.org/10.1371/journal.pone.0024522 